\title{
Accuracy of minor salivary gland biopsy in the diagnosis of Sjögren syndrome
}

\author{
Edelstein $\mathrm{R}^{1}$, Kilipiris $\mathrm{GE}^{1}$, Machalekova $\mathrm{K}^{2}$, Mouzalini $\mathrm{E}^{3}$, Slobodianuk $\mathrm{A}^{4}$, Javorka $\mathrm{V}^{1}$ \\ Department of Stomatology and Maxilofacial Surgery, Faculty of Medicine, Comenius University, \\ and Institute of Oncology St. Elisabeth, Bratislava, Slovakia. rastislav.edelstein@ousa.sk
}

\begin{abstract}
OBJECTIVES: The aim of this study was to find out the correlation and evaluate the accuracy of labial minor salivary gland biopsy as a diagnostic tool in the multidisciplinary management of patients with Sjögren syndrome.

MATERIALS AND METHODS: Patients, referred to our outpatient office between January 2014 and December 2018 from a rheumatologist for biopsy examination, as part of the complex diagnostic plan for suspected Sjögren syndrome, were included in the current study. Each specimen was examined histomorphometrically by the pathologist to calculate the focus score describing the degree of salivary gland inflammatory infiltration.

RESULTS: Fifty patients met the inclusion criteria. From the total number of patients, 39 presented with an established Sjögren syndrome by fulfilling the revised American-European criteria. From those, 27 had a positive lip biopsy. The remaining 12 patients from the total group, who were diagnosed with Sjögren syndrome based on the same criteria, had a negative lip biopsy.

CONCLUSION: The labial minor salivary gland biopsy is a valuable diagnostic tool to establish the diagnosis of Sjögren syndrome. However, a positive biopsy result must always be correlated with all the other diagnostic criteria to prove the exact diagnosis (Tab. 1, Fig. 4, Ref. 49). Text in PDF www.elis.sk KEY WORDS: Sjögren syndrome, keratoconjunctivitis sicca, xerostomia, labial minor salivary glands, biopsy, focal lymphocytic infiltration, focus score.
\end{abstract}

\section{Introduction}

Sjögren syndrome is a systemic, slowly progressive, chronic inflammatory autoimmune disease characterized by a chronic lymphocytic invasion and eventual destruction of exocrine glandular structures, specifically the salivary and lacrimal glands. It is one of the most prevalent autoimmune disorders (1).

Classically, two types have been described:

1. the primary Sjögren syndrome characterized by a combination of keratoconjunctivitis sicca and xerostomia and

2. the secondary Sjögren syndrome, which is defined by a triad of keratoconjunctivitis sicca, xerostomia and an autoimmune disease, usually rheumatoid arthritis, but also systemic lupus erythematosus or scleroderma.

${ }^{1}$ Department of Stomatology and Maxilofacial Surgery, Faculty of Medicine, Comenius University, and Institute of Oncology St. Elisabeth, Bratislava, Slovakia, ${ }^{2}$ Institute of Pathology of Slovak Health University and Institute of Oncology St. Elisabeth, Bratislava, Slovakia, ${ }^{3}$ Military Dental Department 424, Military Hospital, Thessaloniki, Greece, ${ }^{4}$ Department of Dentistry, Oral and Maxillofacial Surgery, Kiev Medical University, Kiev, Ukraine

Address for correspondence: R. Edelstein, MD, Dept of Stomatology and Maxilofacial Surgery, Faculty of Medicine, Comenius University, and Institute of Oncology St. Elisabeth, Heydukova 10, SK-812 50 Bratislava, Slovakia.
As is the case of the majority of autoimmune disorders, the precise aetiology of Sjögren syndrome is unknown. A genetic predisposition involving the major histocompatibility (MHC) locus is likely with individuals sharing haplotypes in the HLADQA_DQB_ region having an increased relative risk, although the diseases are described worldwide. These major histocompatibility haplotypes are hypothesized to result in an aberrant immune response in conjunction with certain environmental triggers. Laboratory studies, as well as some indirect epidemiologic evidence, implicate viruses, including Epstein-Barr virus (EBV) as participants in disease pathogenesis $(2,3)$.

When tissue is damaged/wounded, a series of signalling events activate the immune system, leading to severe inflammatory responses that trigger epithelial-mesenchymal transition, a complex reprogramming process that gradually converts epithelial cells to mesenchymal-like cells, contributing to pathological fibrosis. The fibrotic process is the main pathological feature in many chronic autoimmune diseases, including rheumatoid arthritis, systemic lupus erythematosus and primary Sjögren syndrome (4).

Sjögren has been reported worldwide in adults and more rarely in children, and there appears to be no racial, or geographic bias in incidence. The disorder, however, has a marked predilection for women and similar to SLE, the female:male ratio is approximately $9: 1$. The disease usually presents in middle age but may occur in children as well as the elderly $(5,6)$. 
The study by Liu et al (7) suggested that an X chromosome gene-dose effect might explain the powerful female bias in Sjögren syndrome with mechanisms probably independent of circulating sex hormones.

The spectrum of the disease extends from an organ-specific autoimmune disease to a systemic process with diverse extraglandular manifestations. The hallmark symptoms of Sjögren syndrome are dry mouth and dry eyes. However, clinical features may also include other head and neck manifestations involving the nose, ears, throat, thyroid gland, and systemic symptoms such as neurologic, pulmonary, gastrointestinal and hematologic (8).

Sjögren syndrome is often undiagnosed or misdiagnosed. The symptoms of Sjögren syndrome may mimic those of menopause, drug side effects, or medical conditions such as: lupus, rheumatoid arthritis, fibromyalgia, chronic fatigue syndrome and multiple sclerosis. Because all symptoms are not always present at the same time and because Sjögren syndrome can involve several body systems, physicians sometimes treat each symptom individually and do not recognize that a systemic disease is present (9).

There is a time delay in the diagnosis due to a lack of specific diagnostic tests and the high frequency of sicca in the general population, so the estimated interval between the initial symptoms and diagnosis of the disease is approximately 6 to 10 years (10). While some patients experience a mild discomfort, others suffer debilitating symptoms that greatly impair their functioning.

Sjögren syndrome is treatable. Early diagnosis and consequently a proper treatment may prevent serious complications and greatly improve the quality of life for these patients (11). However, patients with Sjögren syndrome are generally picked up at a late stage in their disease, after the salivary and lacrimal glands are already destroyed, because they are asymptomatic until that time. Unfortunately, at this point only symptomatic treatment can be offered (12).

Although rheumatologists have primary responsibility for managing Sjögren syndrome, patients suspected to have Sjögren syndrome are often referred to an Oral Pathologist for evaluation and biopsy to rule out the disease.

A Sjögren syndrome work-up can include various objective tests, such as Schirmer test, sialometry, injection sialography and scintigraphy that add little to the diagnosis, but provide an information about the degree of ductal and acinar destruction (13). The same information can be obtained from a CT or MRI scan, which will often show internal hypodense areas indicative of ductal ectasia and salivary pooling.

A more focused work-up should be sought to establish a histopathological confirmation. For this purpose, oral labial minor salivary gland biopsy has been traditionally considered the most valuable diagnostic tool for the diagnosis of Sjögren syndrome, especially in patients, who present with inconclusive clinical findings (14). Minor salivary gland biopsy plays an essential role in the diagnosis, stratification, and prognosis of Sjögren syndrome as well as in the dif- ferential diagnosis of the disease (i.e., sarcoidosis, amyloidosis, etc.) (15). Evidence suggests that it has the potential to stratify patients $(16,17,18,19)$ and may have a potential as a biomarker in clinical trials (20).

With all this background, the aim of this study was to discover the accuracy and effectiveness of this diagnostic procedure in the establishment of diagnosis in patients with suspected Sjögren syndrome.

\section{Materials and methods}

Between January 2014 and December 2018, 50 patients were referred to the Outpatient Office of the Department of Periodontology and Oral Medicine, St. Elizabeth Oncologic Clinic and Comenius University in Bratislava, Slovakia with a suspected Sjögren syndrome.

The criteria for patient selection to this study were: i) patients sent from rheumatologists for further examination of suspected Sjögren syndrome and ii) patients with at least one sicca symptom (either xerostomia or xerophthalmia) at the time of presentation. Patients with other established systemic diseases with symptoms similar to Sjögren syndrome and xerostomia as the result of medicaments, radiotherapy to the head and neck region and chemotherapy were excluded from the study.

A complete history and physical evaluation were performed. In addition to lip biopsy, the following diagnostic tools were employed: anti-SSA/Ro or anti- SSB/La antibodies, Schirmer test, ultrasonography and scintigraphy.

Biopsy of the small salivary glands is often a required examination in dental surgery, but its diagnostic value depends largely on the correct indication. Although this is not a particularly burdensome procedure for the patient, the outcome may be many times negative if at least two of the objective international criteria for the suspected diagnosis of SS have not been confirmed (21).

The biopsy specimens were taken from beneath a clinically normal mucosa of the lower lip between the midline and commissure, and 5 to 10 minor salivary glands were removed for examination. Local infiltration with anaesthetic containing vasoconstrictor was applied, followed by a single incision of $1.5-2 \mathrm{~cm}$ vertically to just penetrate epithelium. The linear incision technique is the most frequently performed procedure and is associated with a low rate of complications of less than $1 \%(22,23,24)$. The mi-
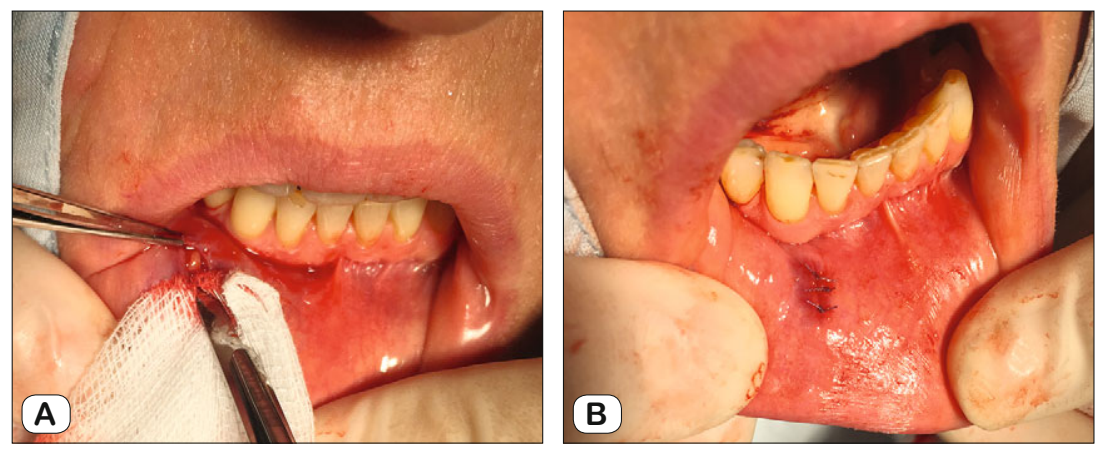

Fig. 1. A. Small Salivary glands visualized during biopsy; B. Situation after suture of wound. 


\section{$454-460$}

nor salivary glands were then removed by blunt dissection, while avoiding sensory nerves (Fig. 1A, B).

The fragments of minor salivary glands were sent for histopathological examination and processed completely according to Sjögren syndrome focus score grading the degree of salivary gland inflammatory infiltration. The focus score of 1 or greater was considered supportive of the diagnosis of Sjögren syndrome. Long term follow-up was introduced with an assessment every 3 months.

In the present study, evaluation of the accuracy of minor salivary gland lip biopsy in the support of Sjögren syndrome diagnosis was performed by comparing the biopsy result (either positive or negative) and the criteria for classification of the disease.

\section{Results}

From the 50 patients meeting the selection criteria, the average age at the time of presentation was 51 years. The oldest patient was 78 years and the youngest 6 years at the time of first examination. Female patients were 37 while the male patients were 13. During a physical examination, patients presented with a wide range of clinical findings including xerostomia, xerophthalmia, difficulty in swallowing, inability to speak continuously for longer than several minutes, altered taste, fissured tongue, red and tender oral mucosa, decreased vision, asymmetric and painless enlargement of major salivary glands.

From the 50 patients included in the study, 39 concluded with an established Sjögren syndrome diagnosis. From those, 27 had a positive lip biopsy and all of them were confirmed to fulfil the revised American-European criteria establishing the diagnosis of the disease. Totally, 12 patients from the group were diagnosed with Sjögren syndrome, based on the above criteria, despite presenting a negative minor salivary gland lip biopsy. All of the patients, 11 in the number, whose diagnostic criteria didn't support the diagnosis of Sjögren syndrome, presented with a negative lip biopsy (Fig. 2).

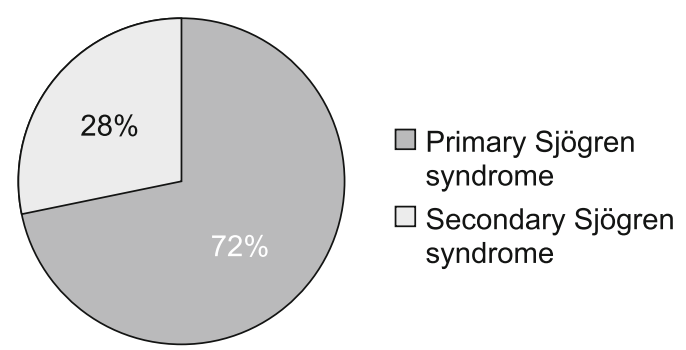

Fig. 3. Distribution of patients with primary and secondary Sjögren syndrome.

Among the 39 patients diagnosed with the disease, 11 were observed with the criteria of secondary Sjögren syndrome, with the most common established connective tissue disorder being rheumatoid arthritis in 7 patients (Fig. 3).

\section{Discussion}

The classification of the disease is currently based on the American-European consensus group classification criteria [American College of Rheumatology (ACR)/European League Against Rheumatism (EULAR)], which include serological tests, clinical findings, and histological examination (Tab. 1). The presence of autoantibodies or a positive minor salivary gland biopsy is mandatory (15).

The diagnosis of primary Sjögren syndrome requires 4 out of the 6 criteria, involving either a positive lip biopsy or positive anti-SSA/Ro or anti-SSB/La. Secondary Sjögren syndrome requires an established connective tissue disease and at least one sicca symptom plus 2 out of 3 objective tests for either xerophthalmia or xerostomia. It should be noted that Sjögren syndrome can also be diagnosed in the absence of sicca symptoms if 3 out of 4 objective tests are positive.

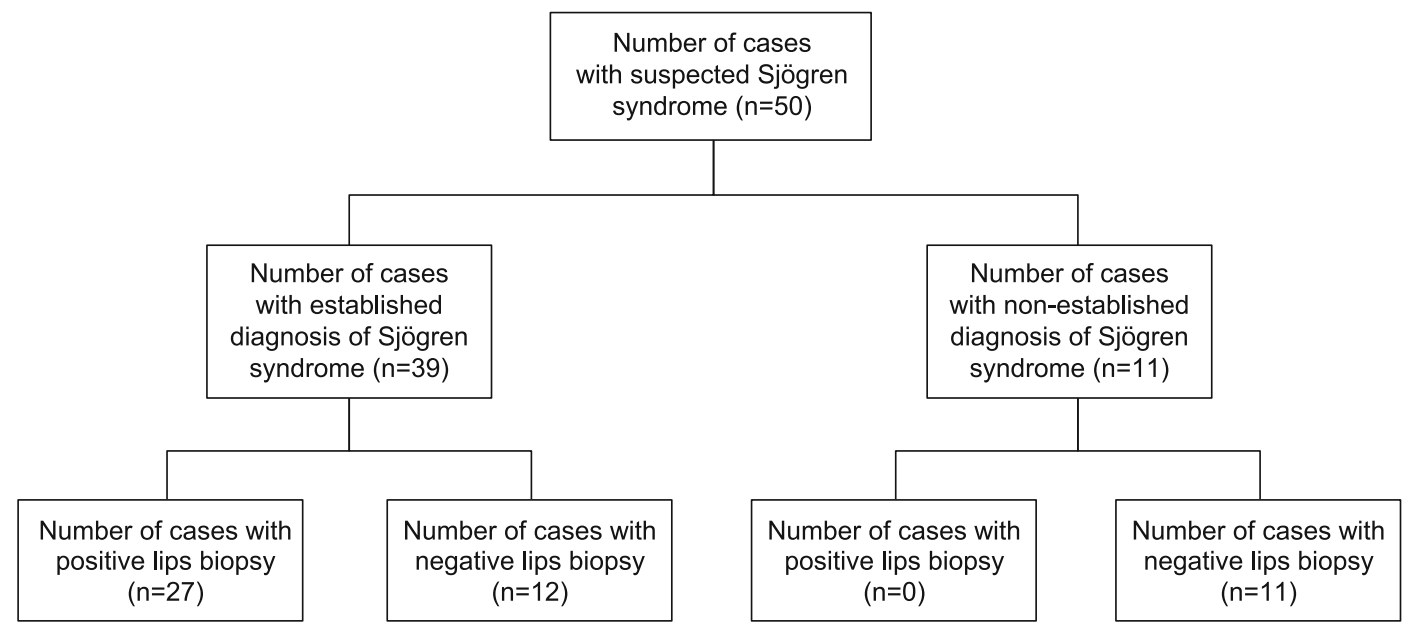

Fig. 2. Flowchart displaying a correlation of labial minor salivary gland biopsy in the establishment of diagnosis of Sjögren syndrome. 
Tab. 1. Revised American-European Criteria for Classification of Sjögren Syndrome.

\begin{tabular}{|c|c|c|c|}
\hline I. Ocular Symptoms (at least one) & $\begin{array}{l}\text { Symptoms of dry eyes for at least } \\
3 \text { months }\end{array}$ & $\begin{array}{l}\text { A foreign body sensation in the } \\
\text { eyes }\end{array}$ & $\begin{array}{l}\text { Use of artificial tears } 3 \text { or more } \\
\text { times per day }\end{array}$ \\
\hline II. Oral symptoms (at least one) & $\begin{array}{l}\text { Symptoms of dry mouth for at least } \\
3 \text { months }\end{array}$ & $\begin{array}{l}\text { Recurrent or persistently swollen } \\
\text { salivary glands }\end{array}$ & $\begin{array}{l}\text { Need for liquids to swallow dry } \\
\text { foods }\end{array}$ \\
\hline III. Ocular signs (at least one) & $\begin{array}{l}\text { Abnormal Schirmer test, (without } \\
\text { anesthesia; } \leq 5 \mathrm{~mm} / 5 \text { minutes) }\end{array}$ & $\begin{array}{l}\text { Positive vital dye staining of the } \\
\text { eye surface }\end{array}$ & \\
\hline IV. Histopathology & $\begin{array}{l}\text { Lip biopsy showing focal } \\
\text { lymphocytic sialoadenitis (focus } \\
\text { score } \geq 1 \text { per } 4 \mathrm{~mm} 2 \text { ) }\end{array}$ & & \\
\hline V. Oral signs (at least one) & $\begin{array}{l}\text { Unstimulated whole salivary flow } \\
\text { ( } \leq 1.5 \mathrm{~mL} \text { in } 15 \text { minutes })\end{array}$ & Abnormal parotid sialography & Abnormal salivary scintigraphy \\
\hline VI. Antibodies (at least one) & $\begin{array}{l}\text { Anti-SSA (Ro) or Anti-SSB (La) } \\
\text { or both }\end{array}$ & & \\
\hline
\end{tabular}

Focal lymphocytic infiltration (FLS) occurs in all organs affected by Sjögren syndrome and is associated with diverse clinical manifestations of the disease. To date, the salivary glands have been the most thoroughly studied among the organs affected by Sjögren syndrome (25).

Salivary glands involved by this condition show a focal lymphocytic pattern of infiltration, in which there are multiple interstitial aggregate foci of inflammatory cells (Fig. 4).

An aggregate focus is defined as a collection of greater than 50 inflammatory cells. The focal lymphocytic infiltrate, including focal aggregates of 50 or more lymphocytes, defined as a focus, that are adjacent to normal appearing acini and the consistent presence of these foci in all or most of the glands in the specimen is the characteristic microscopic feature of Sjögren syndrome in the minor salivary glands. These histopathological changes represent the hallmark of this disorder (26).

The infiltrate should consist predominantly of lymphocytes (Fig 3). Our study showed that the prevalent cells in the minor labial salivary gland infiltrate were those bearing the T-helper phenotype (CD4+). Similar results arose from the large study by Wicheta et al (27), where they studied the role of minor salivary

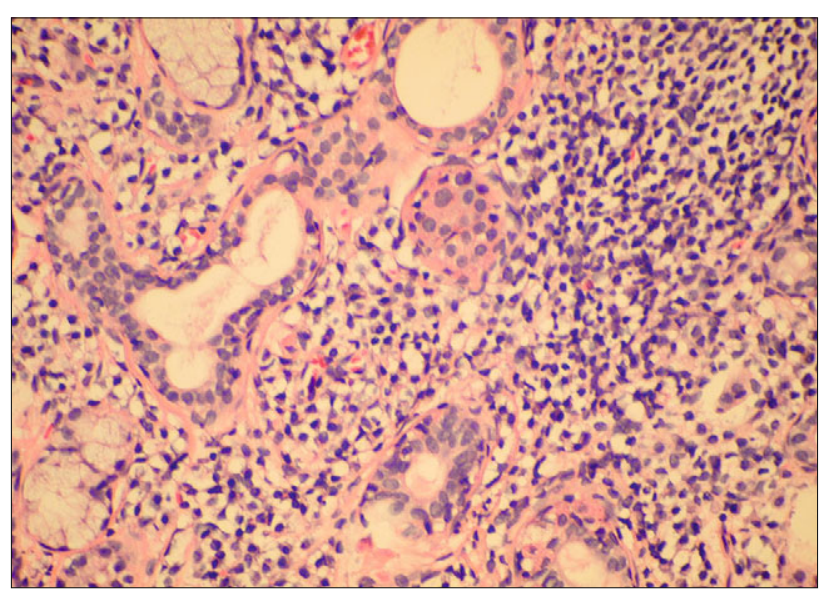

Fig. 4. Microscopic preparation stained with haematoxylin and eosin, showing small salivary glands with lymphocyte infiltrate. gland biopsy in patients with Sjögren syndrome. These T cells also express the adhesion molecule LFA- 1 (lymphocyte function associated molecule) and other T cell markers, such as CD2 and LFA-3, which mediate an antigen independent interaction and are up-regulated after lymphocytic activation. B cells constitute approximately $20 \%$ of the total infiltrating population, while NK cells are rarely observed.

The Sjögren syndrome focus score is a semiquantitative method of grading the degree of salivary gland inflammatory infiltration (28). Histomorphometric analysis is utilized by the pathologist to quantitate the area of salivary gland parenchyma in square millimetres, by counting the number of lymphocytic aggregates. The focus score represents the number of lymphocytic aggregates per 4 square millimetres, and therefore an absolute minimum of 4 square millimetres of salivary gland tissue is required to calculate the focus score. The focus score of 1 or greater is considered supportive the diagnosis of Sjögren syndrome. The focus score can range from 0 to 12 , with the focus score of 12 representing a diffuse glandular effacement by the lymphocytic infiltrate and the score 0 referring to the absence of these cells. The presence of a dense effacing infiltrate should raise concern for possible progression to lymphoma.

The focus score has been validated as a histological index of severity of the salivary gland involvement in Sjögren syndrome. A series of manuscripts have correlated the presence of high focus scores with indices of local or systemic disease activity. The presence of a higher focus score has been found to correlate with acinar damage (29), presence of anti-SSA/B serology (12 times higher among those with focus score $>1$ than among those with focus score $<1$ ), and the presence of specific extra glandular features such as: Raynaud's phenomenon, vasculitis, lymph node or spleen enlargement and leukopenia (30). The focus score $>1$ has also been found to correlate with positive RF serology, high ANA titres and IgG concentrations, the presence of keratoconjunctivitis sicca and low unstimulated salivary flow rates $(31,32)$. More recently, it has been established that a high focus score $(>3)$ has a significant predictive value for the development of non-Hodgkin B cell lymphoma (33).

In our study, the focus score in the group of the examined patients, who had a positive minor salivary gland biopsy, was 
extended from 1 to 6.42 with the highest values seen in patients with primary Sjögren syndrome. It was also pointed out that the focus score cannot separate an early from late disease as chronicity of symptoms and focus score did not show a relationship (29).

Minor salivary gland lip biopsy results report a useful diagnostic value in Sjögren syndrome. They should be carefully addressed in the overall diagnostic procedure due to inconsistencies of sensitivity and specificity.

Whilst the focus score has been proven as a functional diagnostic and prognostic tool, it presents obvious limitations. First, the stability of the focus score in repeated biopsies over a long period of time is not fully established (34). Surprisingly, in $12.6 \%$ of the cases, the second evaluation by trained pathologists led to a diagnosis change (35).

It is possible that widespread cross-institutional failure to apply the focus scoring system in the interpretation of labial salivary gland biopsies may delay the recognition and/or treatment of SS (36). Minor salivary gland infiltration may also be revealed in patients affected by myasthenia gravis, sialolithiasis and other autoimmune disorders not associated with sicca symptoms (37).

In addition, the extent of infiltrate in a lip biopsy using the same methodological approach may vary greatly from gland to gland in a single patient. Further, if the density of infiltrate is severe, the foci may become confluent, hindering focus score determi nation (38).

Whilst the last studies, in particular the correlation between a higher focus score and the development of lymphoma, suggest a stability of the histological lesions over a period of time, this has not been proven in large cohort studies. Moreover, the sensitivity of focus score is reduced in smokers (39). The combination of focus score $>1$ and immunological staining for IgA has been shown to increase the diagnostic specificity for Sjögren syndrome. Indeed, the presence of a focus score $>1$ and quantitative immunohistological staining of $\operatorname{IgA}<70 \%$, had a greater sensitivity and specificity that the focus score alone (40).

Evaluation of a patient with a suspected Sjögren syndrome should include an evaluation of oral and ocular dryness and function. In addition to the history, this may include the performance of a Schirmer test, slit-lamp exam with a vital dye staining, salivary flow rate, and/or nuclear scintigraphic evaluation of the salivary glandular function. An assessment of autoantibodies (ANA, RF, SS-A, and SS-B) should also be performed. Of these, SS-A is probably the most sensitive and specific antibody for Sjögren's but alone it is not diagnostic since it may be present in other autoimmune disorders and may be absent in up to a third of Sjögren cases. The most specific single test is a minor salivary gland (lip) biopsy, which will demonstrate FLS in positive specimens $(41,42,43)$.

Difficulties in MSG biopsy interpretation may arise, because in addition to the FLS, other conditions may also be observed, such as nonspecific chronic sialadenitis (NSCS; i.e., focal or scattered infiltrate of lymphocytes with mild to moderate structure alteration in lobules), chronic sclerosing sialadenitis (i.e., advanced stage of NSCS), granulomatous inflammation, germinal centre (GC) formation, acinar atrophy, interstitial fibrosis, and ductal dilation.
These findings are relatively common and their incidence increase with age (44). If FLS is identified despite these alterations, all foci should be counted to calculate the FS, including foci adjacent to abnormal acini. All the alterations should be stated in the pathology report (45).

The minor salivary gland biopsy is a major variable in the diagnosis of Sjögren syndrome, as long as it is done correctly. Although the majority of the histological studies for the diagnosis of Sjögren syndrome are based on H\&E staining, imunohistochemistry procedures are currently carried out to characterize mononuclear infiltration and obtain additional information about proliferation, migration, antibody secretion, and possible formation of GC. However, in some cases, the minor salivary gland biopsy can be negative. Under such circumstances and if Sjögren syndrome is suspected, anti-Ro antibodies should be present $(46,47)$.

\section{Conclusion}

Minor (labial) salivary gland biopsy is the diagnostic criterion with the highest sensitivity and specificity and should be performed to confirm the diagnosis in all the patients with a suspected Sjögren syndrome, who had negative test results for anti-Ro/ SSA antibodies (48). The sensitivity (63.5-93.7\%) and specificity (61.2-100\%) of this method are high, $>80 \%$ in most studies (22), with a positive predictive value of $95 \%$ and negative predictive value of $92.6 \%$ (49).

The linear incision technique with a collection of at least 4-6 glands or $8 \mathrm{~mm}$ of glands tissue is recommended (21). However, a positive Sjögren syndrome focus score is not diagnostic of Sjögren syndrome by itself, but the results of the biopsy must be correlated with each of the other diagnostic criteria to establish an accurate diagnosis.

Furthermore, the heterogenicity of the measurements might present a potential risk of compromising the combined analysis of different trials. The current interest in designing clinical trials in Sjögren syndrome will therefore require a combined effort of rheumatologists and oral medicine specialists to discuss these aspects and define consensus guidelines on the methodology and the use of the salivary gland biopsy analysis in clinical trials. This increased awareness will help to reduce the time to diagnosis, to direct the treatment from symptomatic, at the exact aetiology behind the disease (tissue specific receptors), and to preserve the health and quality of life of patients with Sjögren syndrome.

\section{References}

1. Mavragani CP, Moutsopoulos HM. Sjögren syndrome. CMAJ 2014; 186 (15): E579-E586.

2. Baer AN, Akpek EK, Alevizos I. 18-21 April 2018, Washington, DC, USA. 14th International Symposium on Sjögren's Syndrome. Clin Exp Rheumatol 2018; 36 Suppl 112 (3): 241-255.

3. Kroese FGM, Haacke EA, Bombardieri M. The role of salivary gland histopathology in primary Sjögren's syndrome: promises and pitfalls. Clin Exp Rheumatol 2018; 36 Suppl 112 (3): 222-233. 
4. Sisto M, Lorusso L, Ingravallo G et al. TGF $\beta 1$-Smad canonical and -Erk noncanonical pathways participate in interleukin-17-induced epithelial-mesenchymal transition in Sjögren's syndrome. Lab Invest 2020; 100 (6): 824-836.

5. Kiadaliri AA, Mohammad AJ, Englund M. Hospitalizations due to systemic connective tissue diseases: Secular trends and regional disparities in Sweden, 1998-2016. Int J Rheum Dis 2018; 21 (11): 1900-1906.

6. Alani H, Henty JR, Thompson NL, Jury E, Ciurtin C. Systematic review and meta-analysis of the epidemiology of polyautoimmunity in Sjögren's syndrome (secondary Sjögren's syndrome) focusing on autoimmune rheumatic diseases. Scand J Rheumatol 2018; 47 (2): 141-154.

7. Liu K, Kurien BT, Zimmerman SL et al. X chromosome dose and sex bias in autoimmune diseases: increased prevalence of 47,XXX in systemic lupus erythematosus and Sjögren's syndrome. Arthritis Rheumatol 2016; 68 (5): 1290-1300.

8. Lee M, Rutka JA, Slomovic AR et al. Establishing guidelines for the role of minor salivary gland biopsy in clinical practice for Sjögren syndrome. J Rheumatol 1998; 25 (2): 247-253.

9. Vitali C, Bombardieri S, Jonsson R et al. Classification criteria for Sjögren's syndrome: a revised version of the European criteria proposed by the American-European Consensus Group. Ann Rheum Dis 2002; 61 (6): 554-558.

10. Mavragani CP, Moutsopoulos HM. The geoepidemiology of Sjögren's syndrome. Autoimmun Rev 2010; 9 (5): A305-A310.

11. Hansen A, Lipsky PE, Dörner T. New concepts in the pathogenesis of Sjögren syndrome: many questions, fewer answers. Curr Opin Rheumatol 2003; 15 (5): 563-570.

12. Feltsan T, Stanko P, Mračna J. Sjögren's syndrome in present. Bratisl Med J 2012; 113 (8): 514-516.

13. Al-Hashimi I. The management of Sjögren's syndrome in dental practice. J Am Dent Assoc 2001; 132 (10): 1409-1417.

14. Pijpe J, Kalk WW, van der Wal JE et al. Parotid gland biopsy compared with labial biopsy in the diagnosis of patients with primary Sjogren's syndrome. Rheumatology 2007; 46 (2): 335-341.

15. Guellec D, Cornec D, Jousse-Joulin S et al. Diagnostic value of labial minor salivary gland biopsy for Sjögren's syndrome: a systematic review. Autoimmun Rev 2013; 12 (3): 416-420.

16. Risselada AP, Kruize AA, Goldschmeding R et al. The prognostic value of routinely performed minor salivary gland assessments in primary Sjogren's syndrome. Ann Rheum Dis 2014; 73 (8): 1537-1540.

17. Theander $\mathbf{E}$, Vasaitis $\mathbf{L}$, Baecklund $\mathbf{E}$ et al. Lymphoid organisation in labial salivary gland biopsies is a possible predictor for the development of malignant lymphoma in primary Sjogren's syndrome. Ann Rheum Dis 2011; 70 (8): 1363-1368.

18. Cornec D, Costa S, Devauchelle-Pensec V et al. Do high numbers of salivary gland-infiltrating B cells predict better or worse outcomes after rituximab in patients with primary Sjogren's syndrome? Ann Rheum Dis 2016; 75 (6): e33.

19. Delli K, Haacke EA, Kroese FG et al. Towards personalised treatment in primary Sjogren's syndrome: baseline parotid histopathology predicts responsiveness to rituximab treatment. Ann Rheum Dis 2016; 75 (11): 1933-1938.

20. Fisher BA, Brown RM, Bowman SJ et al. A review of salivary gland histopathology in primary Sjogren's syndrome with a focus on its potential as a clinical trials biomarker. Ann Rheum Dis 2015; 74 (9): 1645-1650.
21. Stanko P, Galbavý Š, Macák D, Feltsan T, Blahuta R. Minor Salivary Gland Biopsy in Evaluation Algorithm of Sjögren's Syndrome. Česká Stomatologie 2008; 108 (2): 47-51.

22. Fisher BA, Jonsson R, Daniels T et al. Sjögren's histopathology workshop group (appendix) from ESSENTIAL (EULAR Sjögren's syndromestudygroup). Standardisation of labial salivary gland histopathology in clinical trials in primary Sjögren's syndrome. Ann Rheum Dis 2017; 76 (7): 1161-1168.

23. Guellec D, Cornec D, Jousse-Joulin S, Marhadour T, Marcorelles P, Pers JO, Saraux A, Devauchelle-Pensec V. Diagnostic value of labial minor salivary gland biopsy for Sjögren's syndrome: a systematic review. Autoimmun Rev 2013; 12 (3): 416-20.

24. Varela-Centelles P, Seoane-Romero JM, Sánchez-Sánchez M, González- Mosquera A, Diz-Dios P, Seoane J. Minor salivary gland biopsy in Sjögren's syndrome: a review and introduction of a new tool to ease the procedure. Med Oral Patol Oral Cir Bucal 2014; 19 (1): e20-23.

25. Trevisani, VFM, Pasoto, SG, Fernandes, MLMS et al. Recommendations from the Brazilian society of rheumatology for the diagnosis of Sjögren's syndrome (Part I): glandular manifestations (systematic review). Advances in Rheumatology 2019; 59 (58): 1-17.

26. Mandel L, Dehlinger N. Primary biliary cirrhosis and Sjögren's syndrome. J Oral Maxillofac Surg 2003; 61 (11): 1358-1361.

27. Wicheta S, Van der Groen T, Faquin WC, August M. Minor Salivary Gland Biopsy-An Important Contributor to the Diagnosis of Sjögren Syndrome. J Oral Maxillofac Surg 2017; 75 (12): 2573-2578.

28. Chisholm DM., Mason DK. Labial salivary gland biopsy in Sjögren's disease. J Clin Pathol 1968; 21 (5): 656 - 660.

29. Greenspan JS, Daniels TE, Talal N, Sylvester RA. The histopathology of Sjogren's syndrome in labial salivary gland biopsies. Oral Surg Oral Med Oral Pathol 1974; 37 (2): 217-229.

30. Gerli R, Muscat C, Giansanti M et al. Quantitative assessment of salivary gland inflammatory infiltration in primary Sjogren's syndrome: its relationship to different demographic, clinical and serological features of the disorder. Br J Rheumatolo 1997; 36 (9): 969-975.

31. Daniels TE, Cox D, Shiboski CH et al. Associations between salivary gland histopathologic diagnoses and phenotypic features of Sjogren's syndrome among 1,726 registry participants. Arthritis Rheum. 2011; 63 (7): 2021-2030.

32. Haldorsen K, Moen K, Jacobsen H, Jonsson R, Brun JG. Exocrine function in primary Sjogren syndrome: natural course and prognostic factors. Ann Rheum Dis. 2008; 67 (7): 949-954.

33. Risselada AP, Kruize AA, Bijlsma JW. Clinical features distinguishing lymphoma development in primary Sjogren's Syndrome - a retrospective cohort study. Sem Arthritis Rheum 2013; 43 (2): 171-177.

34. Barone F, Campos J, Bowman S, Fisher BA. The value of histopathological examination of salivary gland biopsies in diagnosis, prognosis and treatment of Sjögren's Syndrome. Swiss Med Wkly 2015; 145 : w14168.

35. Costa S, Quintin-Roue I, Lesourd A et al. Reliability of histopathological salivary gland biopsy assessment in Sjogren's syndrome: a multicentre cohort study. Rheumatology 2015; 54 (6):1056-1064.

36. Vivino FB, Gala I, Hermann GA. Change in final diagnosis on second evaluation of labial minor salivary gland biopsies. The Journal of Rheumatology 2002; 29 (5): 938-944. 


\section{$454-460$}

37. Vitali C, Tavoni A, Simi $\mathbf{U}$ et al. Parotid sialography and minor salivary gland biopsy in the diagnosis of Sjögren's syndrome. A comparative study of 84 patients. J Rheumatol 1988; 15 (2): 262-267.

38. Gomes P, Juodzbalys G, Fernandes MH, Guobis Z. Diagnostic Approaches to Sjögren's syndrome: a Literature Review and Own Clinical Experience. J Oral Maxillofac Res 2012; 3 (1): e3.

39. Manthorpe R, Benoni C, Jacobsson L et al. Lower frequency of focal lip sialadenitis (focus score) in smoking patients. Can tobacco diminish the salivary gland involvement as judged by histological examination and anti-SSA/Ro and anti-SSB/La antibodies in Sjögren's syndrome? Ann Rheum Dis 2000; 59: 54-60.

40. Zandbelt MM, Wentink JR, de Wilde PC et al. The synergistic value of focus score and IgA\% score of sublabial salivary gland biopsy for the accuracy of the diagnosis of Sjögren's syndrome: a 10-year comparison. Rheumatology (Oxford) 2002; 41 (7): 819-823.

41. De Vita S, Gandolfo S, Zandonella Callegher S, Zabotti A, Quartuccio $\mathbf{L}$. The evaluation of disease activity in Sjögren's syndrome based on the degree of MALT involvement: glandular swelling and cryoglobulinaemia compared to ESSDAI in a cohort study. Clin Exp Rheumatol 2018; 36 Suppl 112 (3): 150-156.

42. Martel A, Coiffier G, Bleuzen A et al. What is the best salivary gland ultrasonography scoring methods for the diagnosis of primary or secondary Sjögren's syndromes? Joint Bone Spine 2019; 86 (2): 211-217.

43. Baer AN, Walitt B. Update on Sjögren Syndrome and Other Causes of Sicca in Older Adults. Rheum. Dis Clin North Am 2018; 44 (3): 419-436.
44. Daniels TE, Aufdemorte TB, Greenspan JS. Histopathology of Sjögren's syndrome. 41-52. In: Talal N, Moutsopoulos HM, Kassan SS (Eds). Sjögren's syndrome: clinical and immunological aspects. Berlin: Springer-Verlag, 1987.

45. Wicheta S, Van der Groen T, Faquin WC, August M. Discrepancies in interpretation of the minor salivary gland biopsy in the diagnosis of Sjögren syndrome. J Oral Maxillofac Surg 2019; 77 (8): 1628-1635.

46. Pijpe J, Kalk WW, van der Wal JE et al. Parotid gland biopsy compared with labial biopsy in the diagnosis of patients with primary Sjogren's syndrome. Rheumatology (Oxford) 2007; 46 (2): 335-341.

47. Sharma R, Chaudhari KS, Kurien BT et al. Sjögren syndrome without focal lymphocytic infiltration of the salivary glands. J Rheumatol 2020; 47 (3): 394-399.

48. Shiboski CH, Shiboski SC, Seror R. International Sjögren's Syndrome Criteria Working Group, et al. 2016 American College of Rheumatology/European League Against Rheumatism classification criteria for primary Sjögren's syndrome: a consensus and data-driven methodology involving three international patient cohorts. Ann Rheum Dis 2017; 76 (1): 9-16.

49. Giovelli RA, Santos MC, Serrano ÉV, Valim V. Clinical characteristics and biopsy accuracy in suspected cases of Sjögren's syndrome referred to labial salivary gland biopsy. BMC Musculoskelet Disord 2015; $16(30): 1-7$. 